\title{
Magnetic-Field Immunity Examination and Evaluation of Transcutaneous Energy-Transmission System for a Totally Implantable Artificial Heart
}

\author{
Takahiko Yamamoto and Kohji Koshiji \\ Department of Electrical Engineering, Faculty of Science and Technology, Tokyo University of Science, 2641, Yamazaki, Noda, \\ Chiba 278-8510, Japan \\ Correspondence should be addressed to Takahiko Yamamoto, yamamoto@rs.noda.tus.ac.jp
}

Received 1 July 2011; Revised 16 September 2011; Accepted 30 September 2011

Academic Editor: Shuo Wang

Copyright ( $) 2012$ T. Yamamoto and K. Koshiji. This is an open access article distributed under the Creative Commons Attribution License, which permits unrestricted use, distribution, and reproduction in any medium, provided the original work is properly cited.

Transcutaneous energy transmission (TET) is the most promising noninvasive method for supplying driving energy to a totally implantable artificial heart. Induction-heating (IH) cookers generate a magnetic flux, and if a cooker is operated near a transcutaneous transformer, the magnetic flux generated will link with its external and internal coils. This will affect the performance of the TET and the artificial heart system. In this paper, we present the design and development of a coil to be used for a magnetic immunity test, and we detail the investigation of the magnetic immunity of a transcutaneous transformer. The experimental coil, with five turns like a solenoid, was able to generate a uniform magnetic field in the necessary bandwidth. A magnetic-field immunity examination of the TET system was performed using this coil, and the system was confirmed to have sufficient immunity to the magnetic field generated as a result of the conventional operation of induction-heating cooker.

\section{Introduction}

Immediate energy transmission using a cable is an easy method for supplying driving energy to a totally implantable artificial heart [1-5]. However, this method increases the risk of infectious disease and decreases the patient's quality of life (QOL). Transcutaneous energy transmission (TET) is the most promising non-invasive method of energy transfer [3$5]$.

If an artificial heart stops because of malfunctioning of the TET system, the consequences are fatal. Therefore, according to the Pharmaceutical Affairs Act in Japan, an evaluation of electromagnetic compatibility (EMC) is indispensable [5]. The EMC requirements for an artificial heart system state that it is necessary to reduce the electromagnetic interference (EMI) and electromagnetic susceptibility (EMS) while maintaining performance. However, even if the examination satisfies the Pharmaceutical Affairs Act, the safety of the system is not necessarily guaranteed from the viewpoint of EMC. Recently, there have been some reports on the malfunctioning of medical devices such as pacemakers that was caused by magnetic-field leakage from inductionheating $(\mathrm{IH})$ cookers [6]. However, EMS evaluation that assumes such an event is not currently obligatory for medical equipment.

A transcutaneous transformer transmits driving energy to an artificial heart implanted inside the body by using electromagnetic induction between two coils inside and outside the body. IH cookers generate a magnetic flux, and if a cooker is operated near a transcutaneous transformer, the magnetic flux generated will link with the transformer's external and internal coils [7]. This can affect the performance of the TET system and the artificial heart system. Hence, it is necessary to investigate the magnetic-field immunity of the TET system. In this paper, we report on the design and development of a coil for the magnetic immunity test, and we detail the investigation of the immunity of transcutaneous transformers. 


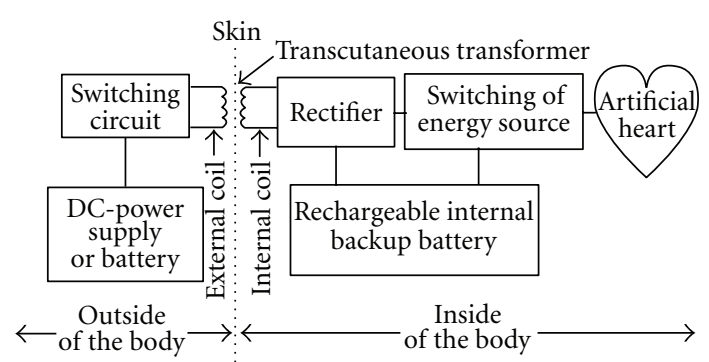

FIGURE 1: Block diagram of TET system.

\section{TET System}

The TET system allows the noninvasive transmission of energy to the inside of the body. Figure 1 displays a block diagram of the TET system. Outside the body, DC electric power that is supplied by a stabilized DC power supply or an external rechargeable battery is converted into highfrequency $(300 \mathrm{kHz})$ AC electric power by a push-pull-type inverter circuit. The AC electric power is transmitted to the inside of the body through the transcutaneous transformer, as shown in Figure 2(a). The transcutaneous transformer consists of an external coil and an internal coil, of nine turns each, using Litz wire. Two primary coils outside the body are closely wound in the toroidal-type ferrite. To detach a coil outside the body easily, the ferritic core combined two ferrites of the character type $\mathrm{C}$. The half of the annulus ring of the coil that is inside the body (nine turns) was buried under the hypodermis, and the remaining half was wrapped with the skin and thrust out like an arch (Figure 2(b)). Figure 2(c) shows an installation of the passing skin transformer on a goat.

Inside the body, AC electric power is converted into DC power in order to drive the artificial heart actuator and charge the rechargeable internal backup battery.

\section{Design of the Coil for the Magnetic Immunity Test}

3.1. Deciding the Magnetic-Field Immunity Examination Frequency. The magnetic-field leakage to the outside of the IH cooker was measured, and the bandwidth at which the magnetic-field immunity test should be carried out was decided on the basis of this result. The magnetic field around the IH cooker (near field) when heating a water-filled pan with the maximum output $(1.4 \mathrm{~kW})$ of the cooker was measured using a near-magnetic-field probe (Hewlett Packard, 11941A, CA, USA) and a spectrum analyzer (Anritsu, MS2661C, Kanagawa, Japan). The distance from the IH cooker was $35 \mathrm{~cm}$ because it was assumed that the patient was working on the counter in the vicinity of the IH cooker.

Figure 3 shows the results of the magnetic-field leakage from the IH cooker. The exciting frequency was $27 \mathrm{kHz}$; the magnetic-field leakage at this frequency was $128 \mathrm{~dB} \mu \mathrm{A} / \mathrm{m}$, and it decreased as the frequency increased. The maximum frequency in the leakage field from the $\mathrm{IH}$ cooker was $1 \mathrm{MHz}$.

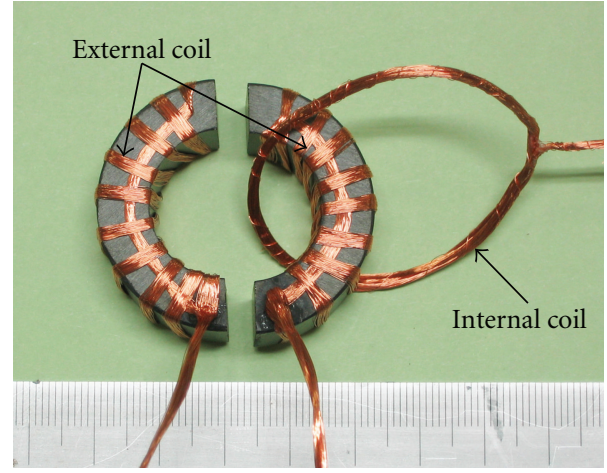

(a) Composition of transcutaneous transformer

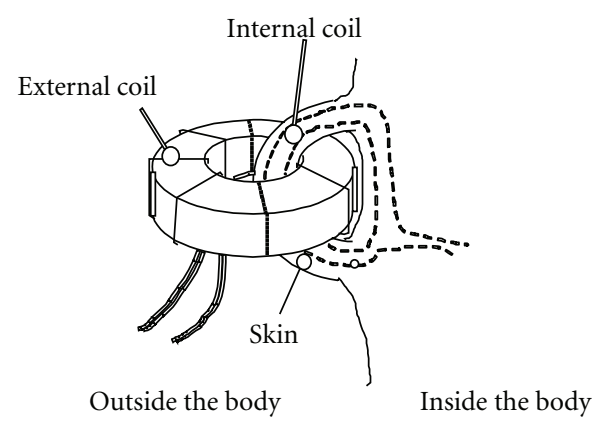

(b) Illustration of internal coil embedding and the transformer wearing

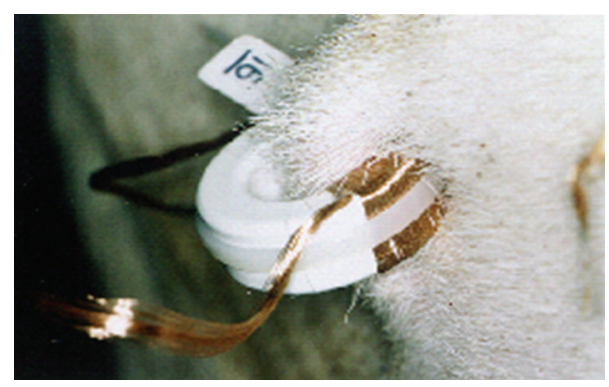

(c) Goat wearing transcutaneous transformer

FIgURE 2: Transcutaneous transformer.

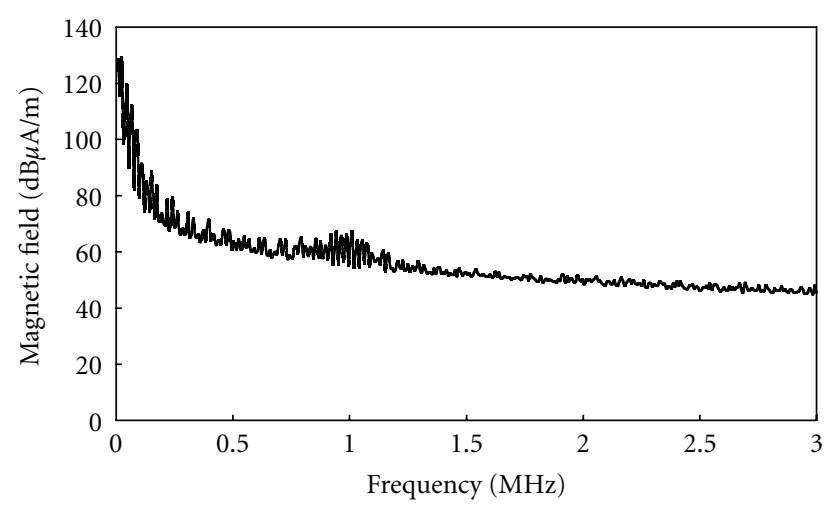

FIGURE 3: Magnetic field from IH cooker (near field). 


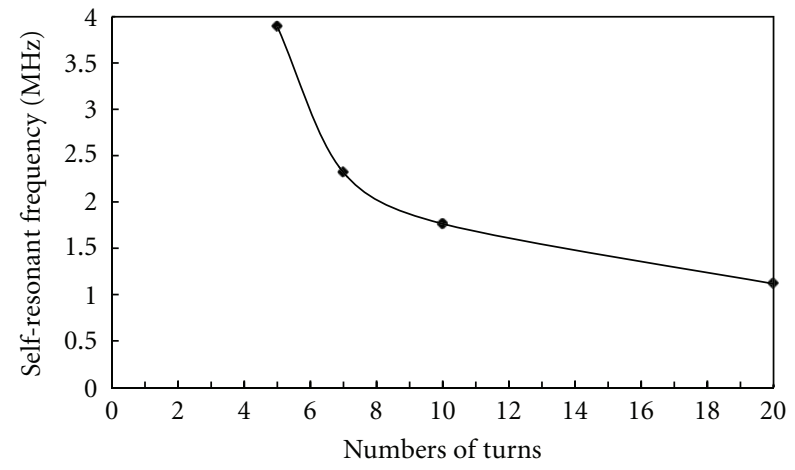

FIGURE 4: Self-resonant frequency of experimental coil (measured).

Therefore, we chose a test frequency range from $1 \mathrm{kHz}$ to $3 \mathrm{MHz}$.

3.2. Fabrication of Test Coil for Trial Purposes. A coil is used to generate the magnetic field in the examination of the magnetic-field immunity. In this test, the coil must be able to generate a uniform magnetic field within the evaluation bandwidth. According to IEC 61000-4-3, the immunity standard for radiated radiofrequencies, the uniform field area of the immunity test must be within the range from 0 to $+6 \mathrm{~dB}$. Therefore, in this paper, the field is considered to be uniform when the fluctuation band of the generated magnetic field is within $6 \mathrm{~dB}$.

A Helmholtz coil can generate a uniform magnetic field. However, the stray capacitance between the winding lines is large because the wire of the coil is concentrated. The self-resonant frequency of the coil decreases, and there is a possibility that the magnetic field cannot be generated in the necessary bandwidth. Therefore, we selected a solenoid coil that increased the self-resonant frequency of the coil for the examination and that was able to generate a uniform magnetic field.

The required power supply, which was generated by increasing the number of coil turns, is low, and therefore, the immunity test can be performed easily and at a low cost. However, the self-resonant frequency decreases with an increasing number of turns. Therefore, the optimal number of coil turns is defined as the maximum number of turns for which the self-resonant frequency of the coil increases more than the bandwidth of the test.

A solenoid coil was fabricated for a cubic with a length of $1 \mathrm{~m}$ for the TET system. Figure 4 shows the relation between the number of turns and the self-resonant frequency. The self-resonant frequency of the coil with five turns was $3.79 \mathrm{MHz}$, and it rose more than $3 \mathrm{MHz}$ of the maximum frequency of the test. It was thus decided that five turns was the appropriate number of turns for the magnetic-field immunity test coil.

Next, the uniform magnetic field of the prototype coil was evaluated. The inside of the coil side was assumed to be the $x$ - and $y$-axes, and the vertical direction on the loop side was assumed to be the $z$-axis. The magnetic field generated in the coil by using a signal generator (ROHDE and SCHWARZ,

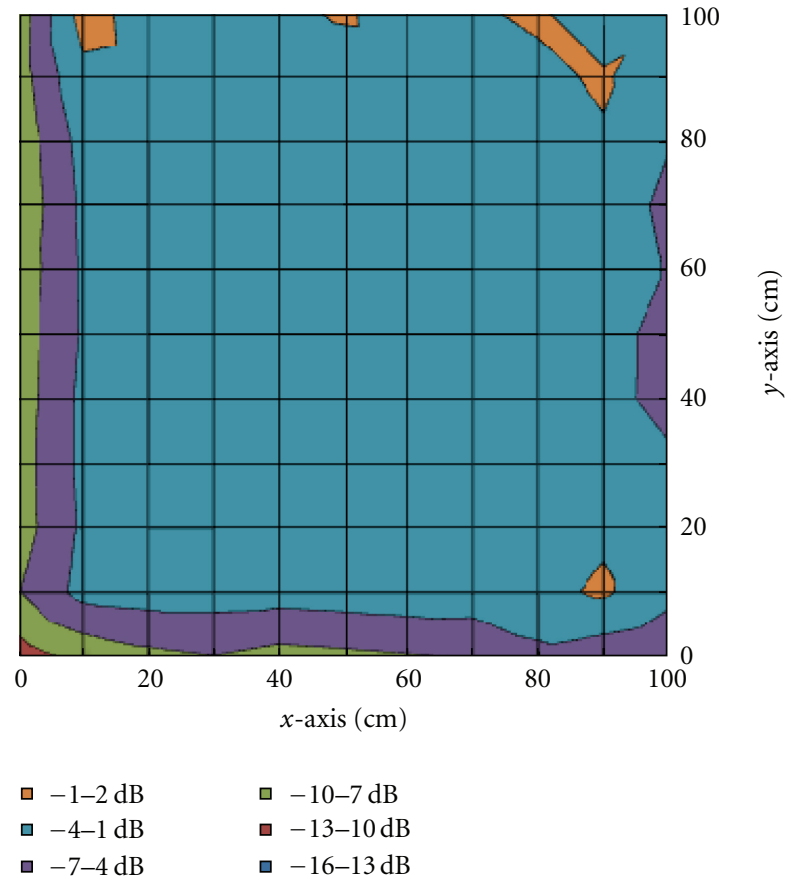

FIGURE 5: Magnetic-field distribution in $x y$-plane.

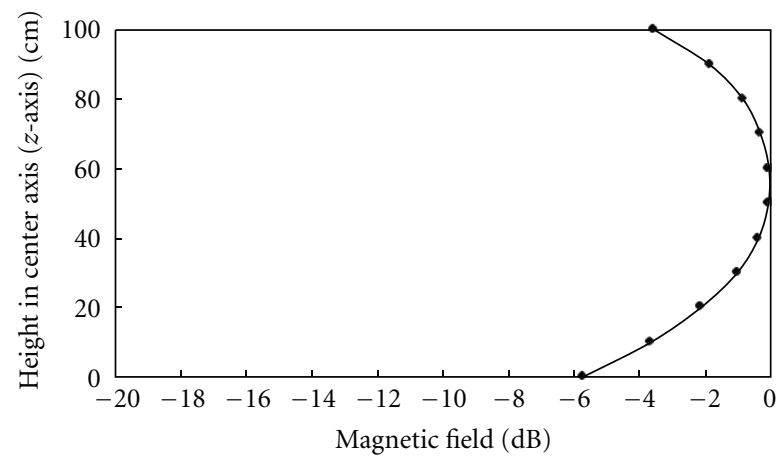

FIGURE 6: Magnetic-field distribution along $z$-axis (measured).

SMY01, Munich, Germany) and a power amplifier (NF Corporation 4025, Saitama, Japan) when the frequency was assumed to be $30 \mathrm{kHz}$ (the excitation frequency of the $\mathrm{IH}$ cooker) was measured. The magnetic field was measured by using the near-field probe and the spectrum analyzer. Figure 5 shows the magnetic-field strength distribution in the $x y$-plane on the top of the solenoid coil $(z=100 \mathrm{~cm})$. The uniformity in the magnetic field in the area of a square with sides of length $0.8 \mathrm{~m}$ on the loop side was $3.3 \mathrm{~dB}$, which complied with the IEC61000-4-3 standard. The magnetic-field strength distribution in the axial direction of the solenoid coil is shown in Figure 6. The magnetic-field uniformity along the $z$-axis was $5.6 \mathrm{~dB}$, which also complied with the standard.

Therefore, the prototype coil was able to generate a uniform magnetic field in the required bandwidth of $3 \mathrm{MHz}$ when wound with five turns like a solenoid. The uniformity 


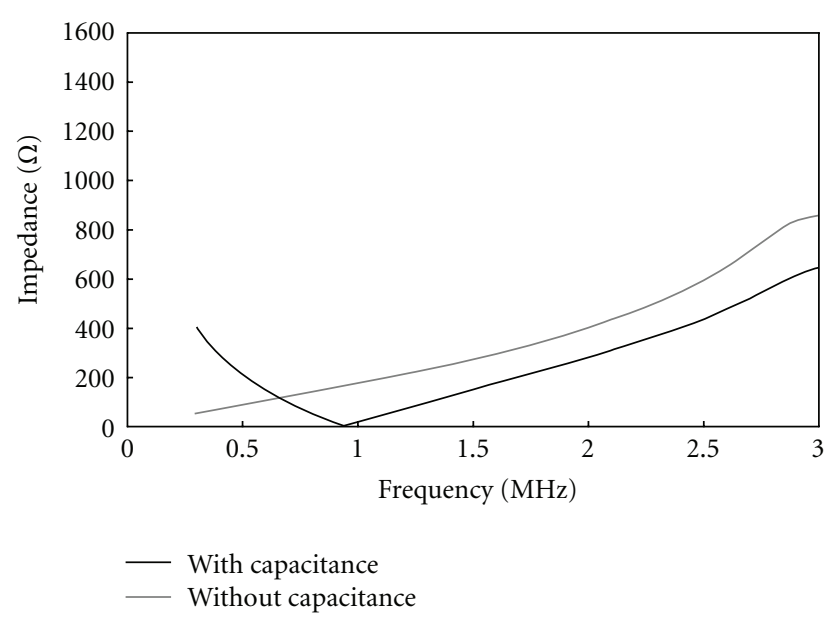

Figure 7: Absolute value of the impedance of the coil.

of the generated magnetic field adhered to the standard defined by IEC61000-4-3.

3.3. Reduction in Coil Impedance. The voltage of the coil is given by expression (1), and it increases in proportion to frequency when a constant current flows into the coil. The reactance of the coil is proportional to the frequency:

$$
V=j \omega L I .
$$

The power-supply voltage necessary for the examination of the magnetic-field immunity increases at high frequencies, and the required test equipment becomes larger in scale. However, the power-supply facility should be as small as possible to ensure the easy examination. To achieve this, the necessary voltage can be decreased by connecting a capacitor in the series; this decreases the synthetic impedance to the coil. The resonance frequency when the inductance $L$ and capacitance $C$ are connected in the series is given by expression (2), and the capacitance is defined by expression (3):

$$
\begin{aligned}
& f=\frac{1}{2 \pi} \sqrt{L C}, \\
& C=\frac{4 \pi^{2} f^{2}}{L} .
\end{aligned}
$$

The examination standard of IEC dictates that the frequency be examined at $1 \%$ intervals. When the examination is done based on this standard, the number of test frequency points is $\log _{1.01} 3000=805$ in the frequency range from $1 \mathrm{kHz}$ to $3 \mathrm{MHz}$ (3000 times). A device that switches the capacitor automatically might be needed. This will be addressed in our future work.

As one example, Figure 7 shows the absolute value of the impedance of the coil and the absolute value of the impedance when a capacitor of $C=1000 \mathrm{pF}$ and the coil are connected in series. The absolute value of the reactance becomes $0 \Omega$ at $960 \mathrm{kHz}$ when a capacitor of $C=1000 \mathrm{pF}$ is inserted, and the impedance can be assumed to be minimal.

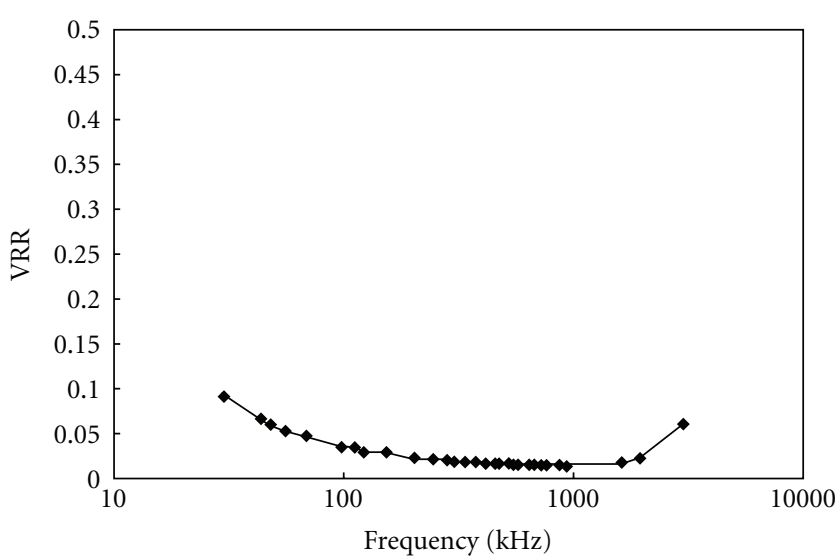

FIGURE 8: Voltage reduction ratio when the capacitor is inserted (measured).

The impedance of the coil can be kept low by sweeping the frequency of the current flowing in the coil while changing the capacitor connected in series to the coil. The voltage reduction ratio (VRR) is defined in expression (4) as follows:

$$
\operatorname{VRR}=\frac{V_{\text {in }}}{V_{L}},
$$

where $V_{\text {in }}$ is the input power-supply voltage and $V_{L}$ is the voltage in the coil. Figure 8 displays the characteristic VRR and frequency when the capacitor determined using expression (3) and the coil are connected in series. The voltage required is $9.9 \mathrm{~V}$ when the capacitance is inserted, and it is possible to decrease this voltage by $1.5 \%$, although a voltage of $643 \mathrm{~V}$ is necessary at $940 \mathrm{kHz}$ for a flow current of $4 \mathrm{~A}$ in the coil.

\section{Magnetic-Field Immunity of the TET System}

The magnetic-field immunity of the TET system was examined using a prototype solenoid coil with five turns. The TET system was set up for the examination in the center part of the coil, and a current of 3.5 A flowed into the coil. The induced voltage in the external and internal coils of the TET system was measured with an oscilloscope (Yokogawa, DL1640, Tokyo, Japan). The generated magnetic field was 140 $\mathrm{dB} \mu \mathrm{A} / \mathrm{m}$. This value was $12 \mathrm{~dB}$ stronger than the maximum magnetic-field strength of $128 \mathrm{~dB} \mu \mathrm{A} / \mathrm{m} 35 \mathrm{~cm}$ above the IH cooker with the water-filled pan, as shown in Figure 3. Figures 9 and 10 show the voltage induced in the external and internal coils of the TET system when the test frequency was assumed to be $30 \mathrm{kHz}$, which was the exciting frequency of the IH cooker. The voltages induced in the external and internal coils were $0.007 \mathrm{~V}$ and $0.079 \mathrm{~V}$, respectively. It can be said that these voltage values are very smaller than the voltage rating of the artificial heart system $(24 \mathrm{~V})$; therefore, the TET system has sufficient magnetic immunity. 


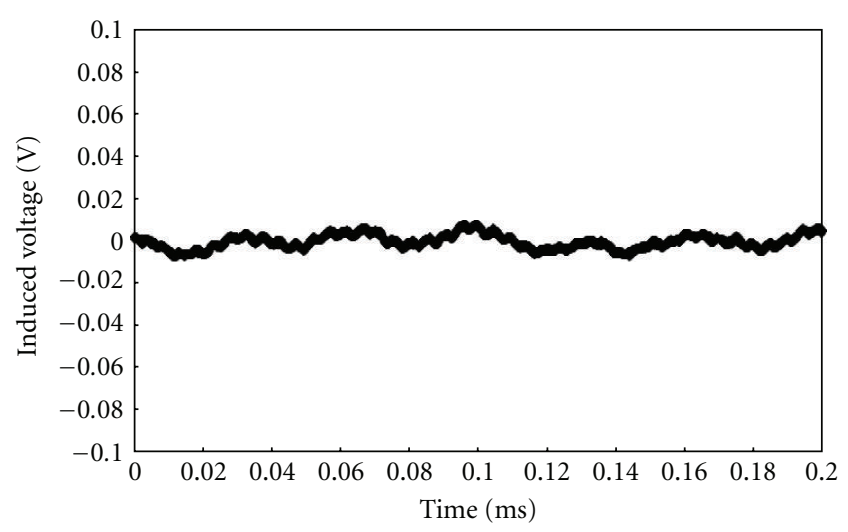

FIGURE 9: Induced voltage in external coil (measured).

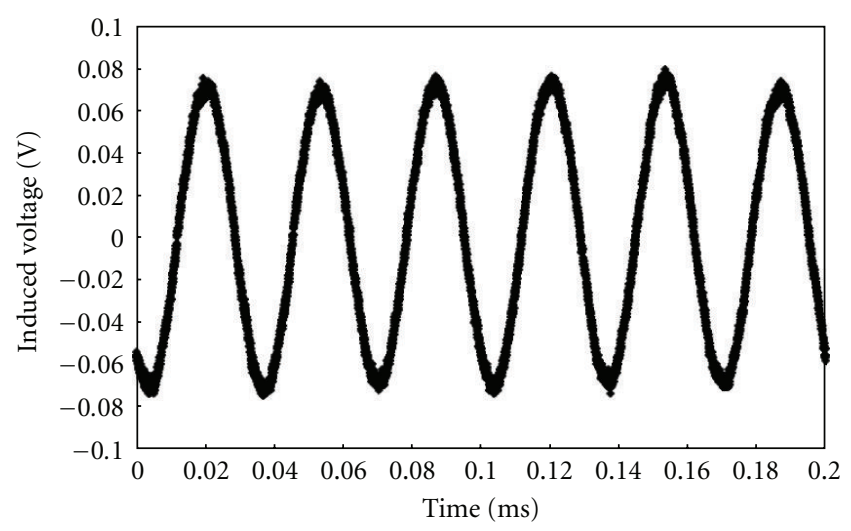

FIgURE 10: Induced voltage in internal coil (measured).

\section{Conclusion}

In this paper, we have reported on the design and development of a coil for the magnetic immunity test. This test is an important factor in guaranteeing the safety of TET systems for totally implantable artificial hearts or other medical devices. In addition, we detailed our investigation of the immunity of a transcutaneous transformer.

The results showed that an experimental coil with five turns like a solenoid was able to generate a uniform magnetic field in the necessary bandwidth. This uniformity of the generated magnetic field complied with the standard detailed in IEC61000-4-3. In addition, the power supply necessary for the examination could be reduced by inserting capacitors in series with the coil. Moreover, the magneticfield immunity examination of the TET system was executed using the coil, and it was confirmed that the TET system had sufficient immunity to the magnetic field produced by the conventional usage of an $\mathrm{IH}$ cooker.

The high-frequency magnetic-field immunity examination proposed and discussed in this paper is important for the evaluation of equipment for home medical care, as there is a risk of exposure to a magnetic field while using such equipment. Therefore, the examination presented here is applicable not only for the TET system but also for evaluating the reliability of many other medical devices.
In future work, we will investigate the magnetic-field immunity of the TET system in other situations involving large magnetic fields and near power stations.

\section{References}

[1] Y. Yokoyama, O. Kawaguchi, T. Shinshi, U. Steinseifer, and S. Takatani, "A new pulse duplicator with a passive fill ventricle for analysis of cardiac dynamics," Journal of Artificial Organs, vol. 13, no. 4, pp. 189-196, 2010.

[2] K. Nawata, T. Nishimura, S. Kyo et al., "Outcomes of midterm circulatory support by left ventricular assist device implantation with descending aortic anastomosis," Journal of Artificial Organs, vol. 13, no. 4, pp. 197-201, 2010.

[3] T. Yamamoto, K. Koshiji, K. Tsukahara et al., "An externallycoupled transcutaneous energy transmission system for totally implantable artificial hearts-detection of abnormal coupling caused by misalignment and air gap in the ferrite core junction of the transcutaneous transformer," Transaction of Japanese Society for Medical and Biological Engineering, vol. 43, no. 2, pp. 261-267, 2005.

[4] T. Hongo, T. Yamamoto, H. Aoki et al., "Investigation on energy transmission efficiency of externally-coupled transcutaneous energy transmission system (ectets) miniaturized by using higher switching frequency for a totally-implantable artificial heart (tiah)," in Proceedings of the American Society for Artificial Internal Organs 54th Annual Conference, p. 49, 2008.

[5] T. Yamamoto, K. Koshiji, Y. Nawa et al., "Transcutaneous energy transmission system for a totally-implantable artificial heart in case using external battery," in Proceedings of the World Congress on Medical Physics and Biomedical Engineering, pp. 3026-3029, 2006.

[6] T. Nagatomo, H. Abe, R. Kohno et al., "Electromagnetic interference with a bipolar pacemaker by an induction heating (IH) rice cooker," International Heart Journal, vol. 50, no. 1, pp. 133137, 2009.

[7] T. Yamamoto, K. Koshiji, A. Homma, E. Tatsumi, and Y. Taenaka, "Improvement in magnetic field immunity of externallycoupled transcutaneous energy transmission system for a totally implantable artificial heart," Journal of Artificial Organs, vol. 11, no. 4, pp. 238-240, 2008. 

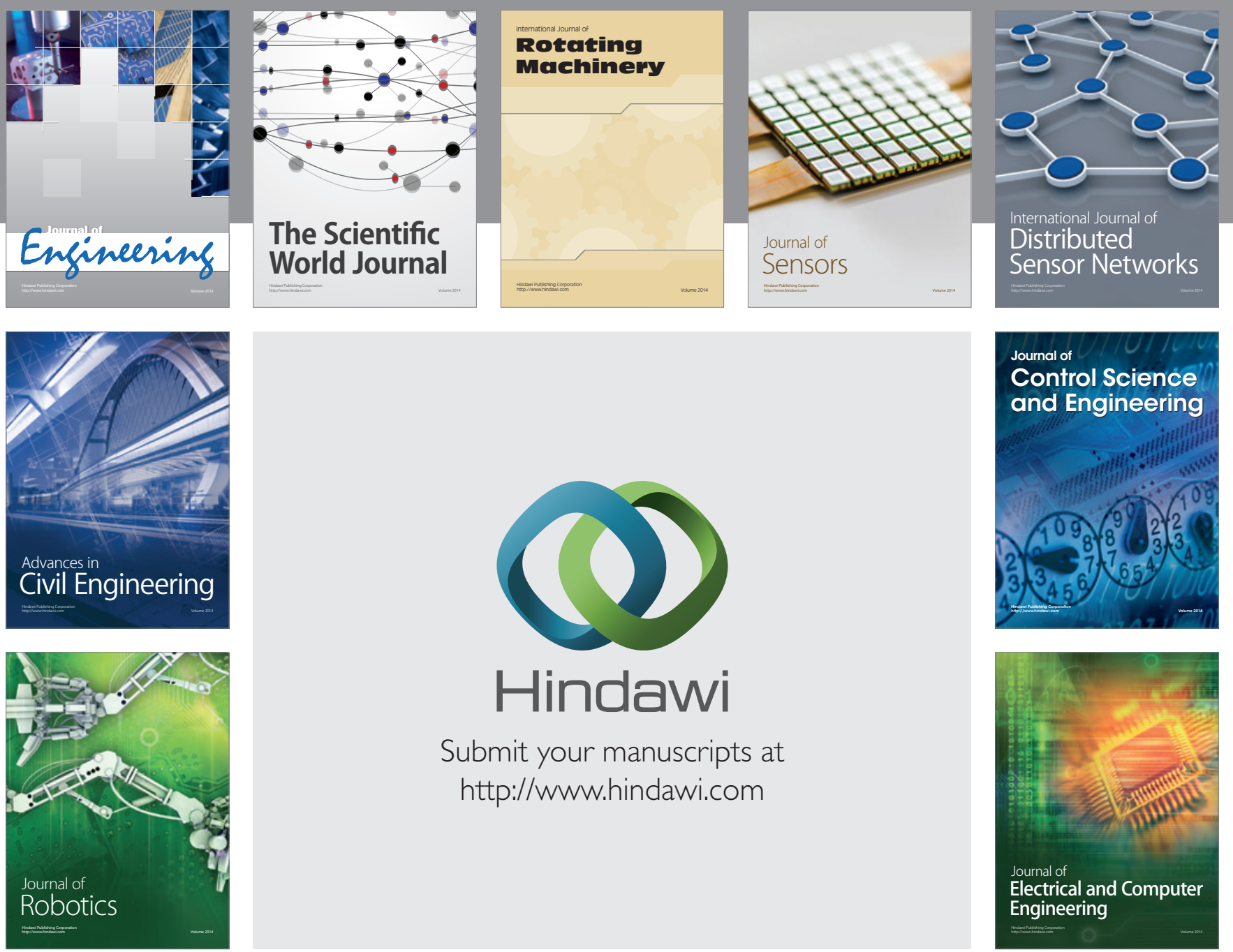

Submit your manuscripts at

http://www.hindawi.com
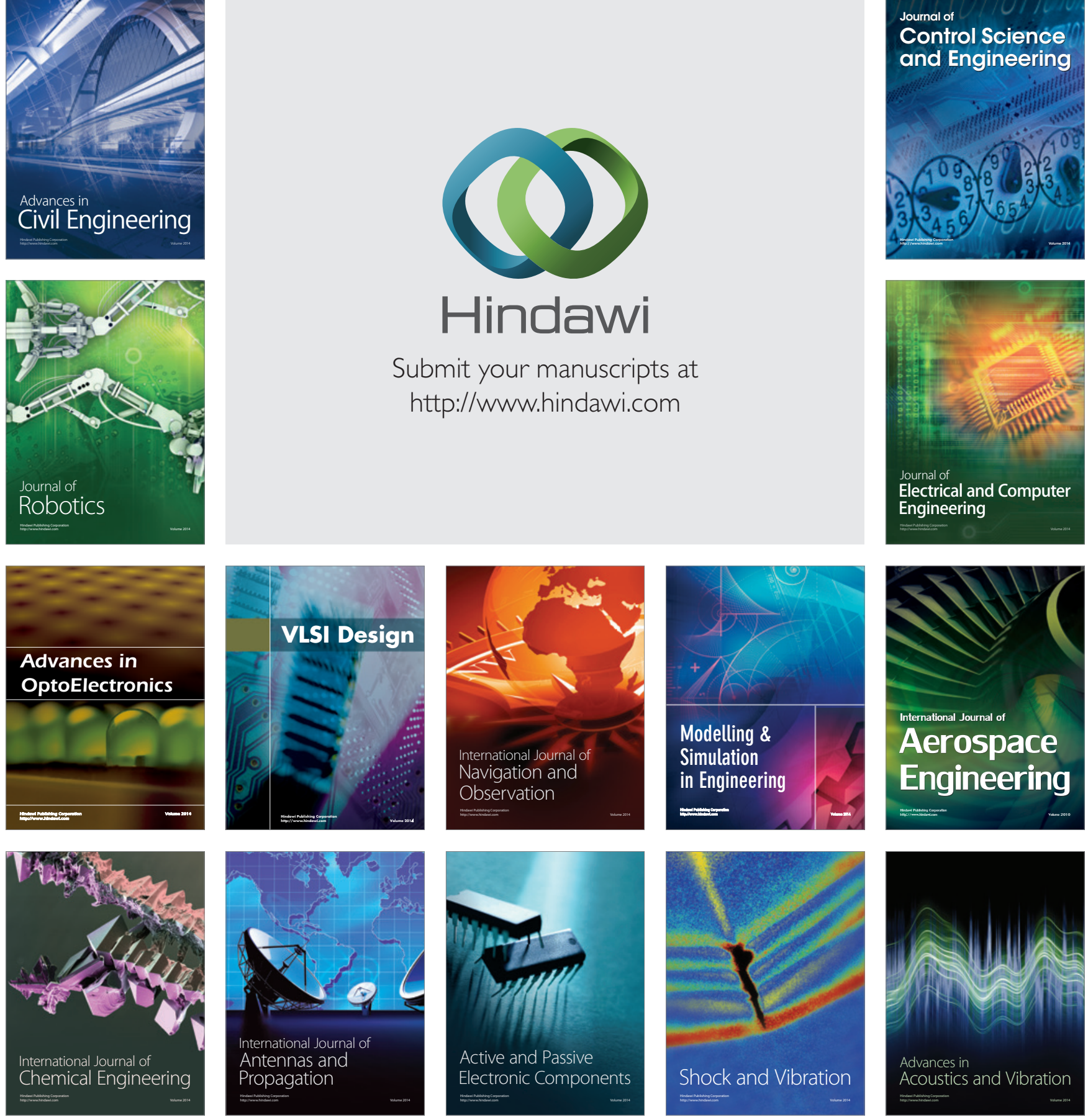